\title{
Spiral arms in near-infrared bands ${ }^{\star}$
}

\section{Broad- and narrow-band NIR photometry}

\author{
P. A. Patsis ${ }^{1,2,3}$, Ph. Héraudeau ${ }^{3}$, and P. Grosbøl ${ }^{4}$ \\ 1 Research Center for Astronomy, Academy of Athens, 14 Anagnostopoulou St., 10673, Athens, Greece \\ 2 Astronomisches Rechen-Institut Heidelberg, Mönchhofstrasse 13, 69120 Heidelberg, Germany \\ 3 Max-Planck Institut für Astronomie, Königstuhl 17, 69117 Heidelberg, Germany \\ 4 European Southern Observatory, Karl-Schwarzschild-Str. 2, 85748 Garching, Germany
}

Received 31 October 2000 / Accepted 15 February 2001

\begin{abstract}
We investigate the contribution of $\mathrm{Br} \gamma$ and $\mathrm{H}_{2}$ emission due to young objects in the arms of spiral galaxies observed in the $K^{\prime}$ filter. Out of a sample of disk galaxies for which we obtained deep surface photometry in broad- and narrow-band near-infrared filters, we selected two grand design spirals (NGC 5861, NGC 7412), which clearly have sharp knots along their arms both in optical and NIR images. For these galaxies we estimate the amount of light coming from $\mathrm{Br} \gamma$ and $\mathrm{H}_{2}$ emission and we conclude that it represents only a few percent of the observed $K^{\prime}$ light. For comparison we used the spiral galaxy NGC 4603, which has high recessional velocity. In this case the emission lines we study are practically shifted out of the narrow-band filter. Comparing its flux with what we found in the two former cases, we conclude that a major contribution from young objects in $K^{\prime}$ comes from continuum radiation which in the arm regions can amount to $20 \%$.
\end{abstract}

Key words. galaxies: photometry - galaxies: spiral - galaxies: structure - infrared: galaxies

\section{Introduction}

A very important parameter for understanding the dynamics of spiral structure in galaxies is the amplitude of the spiral perturbation. The mean amplitude varies for galaxies along the Hubble sequence but significant radial variations within a galactic disk are also observed. Typical relative amplitudes of the main spiral arms in $K^{\prime}$ are in the range of $0.1-0.2$. The strength of the spiral perturbation determines the region of the galactic disks where, in the framework of the Density Wave Theory, linear models can be applied to explain the observed structures (Lin et al. 1969; Bertin et al. 1989), or where nonlinear effects become important (Contopoulos \& Grosbøl 1986, 1988; Patsis et al. 1991). Changes of the pitch angle of a spiral, the break of its symmetric morphology or bifurcations of the arms are typically associated with major dynamic resonances in the disk (Patsis et al. 1997). The intensity and even the existence of such features is directly related to the amplitude of the spiral.

Send offprint requests to: P. A. Patsis,

e-mail: ppatsis@cc.uoa.gr

* Based on observations collected at the European Southern Observatory, La Silla, Chile.
The lifetime of a spiral pattern and the amplification mechanisms (Toomre 1981) to sustain it depend also on the amplitude of the perturbation itself. We note that the orbital behavior and the general dynamics of stellar disks are considerably different for weak and strong perturbations (Patsis et al. 1991).

To determine the properties of spiral density perturbations from observations, one needs to measure the distribution of the old stellar disk population which constitutes the major fraction of the mass. The near infrared $K^{\prime}$ band around $2.1 \mu \mathrm{m}$ is most suitable for such studies since attenuation by dust is insignificant. Also, the energy distribution of the old stars peaks in the near-infrared. Thus, it is less sensitive to contribution from young stars. However, exactly how much young objects contribute in this band remains an open question (Rix \& Rieke 1993; Rhoads 1996; Chiosi \& Vallenari 1996; Grosbøl \& Patsis 1998). The analysis of five spiral galaxies (Grosbøl \& Patsis 1998) shows that the exponential scale length of the disk in the inter-arm regions is smaller than in spiral arms. This could be explained by an increasing fraction of extreme Pop. I objects in the outer parts of the arms. Further, many knots along the arms are observed in $K^{\prime}$. These facts suggest that a significant fraction of the light in the spiral arms originates from young objects. 
Thus, it is mandatory to estimate the importance of population variations in spiral arms and correct for them in order to perform a detailed comparison between observed galaxies and dynamic models.

Radiation from young objects is expected to be present in the $K^{\prime}$ band (Grosbøl \& Patsis 1998). The knots on the arms mentioned above are the first candidates to be examined for this. Their confined extent indicates a dynamically unevolved, and thus young, population. Dynamical processes, like stellar orbit diffusion, would dissolve stellar groups in less than $50 \mathrm{Ma}$ (Wielen 1977).

In order to estimate changes of $M / L$ due to population effects one has to examine the contribution of three major sources: HII regions, molecular hydrogen and red supergiants. Our main interest in studying the $K$ band photometry is to estimate the mass distribution in the galactic disks. For this purpose the knowledge of the contribution of light coming from young objects included in NIR-band filters is essential. In this paper we examine the $\mathrm{Br} \gamma, \mathrm{H}_{2}$ and continuum contribution from young objects by observing three galaxies using the appropriate narrowband filters.

\section{The observed galaxies}

From our sample we selected three galaxies described in Table 1, which are classified as normal in the Revised Shapley-Ames Catalog (RSC) (Sandage \& Tammann 1981). The first two have relatively low systemic velocities. This is necessary to ensure that the emission lines are fully in the narrow-band filter. Optical images of these two galaxies showing their spiral structure are given in Fig. 1. NGC 5861 is a grand design galaxy with typical interarm features, while NGC 7412 has broad arms with faint extensions and bifurcations. The main spiral pattern is asymmetric in this case. We obtained for these galaxies narrow-band near-infrared surface photometry in BP8 $(\mathrm{Br} \gamma)$ and BP5 $\left(\mathrm{H}_{2}\right)$ filters and we compared it with the $K^{\prime}$ surface photometry, in order to estimate the contribution of the $\mathrm{Br} \gamma$ and $\mathrm{H}_{2}$ line emissions. The recessional velocity of the third galaxy, NGC 4603, is larger and therefore the emission lines are shifted out of the narrow-band filter. This galaxy is used to compare the narrow-band filter photometry with that of the two former galaxies, as described in Sect. 5.1.

Table 1. The observed galaxies. The morphological type is taken from RSC; the diameter $D_{25}$, the ratio $R_{25}$, and the Heliocentric velocity from optical lines from RC3 (de Vaucouleurs et al. 1991)

\begin{tabular}{ccccc}
\hline Galaxy & Type & $D_{25}$ & $R_{25}$ & $v_{\text {opt }}\left(\mathrm{km} \mathrm{s}^{-1}\right)$ \\
\hline NGC 5861 & Sc(s)II & $33^{\prime} 0$ & 1.8 & 1826 \\
NGC 7412 & Sc(s)I-II & $3{ }^{\prime} \cdot 9$ & 1.3 & 1716 \\
NGC 4603 & Sbc(s)I-II & $3 \cdot 4$ & 1.4 & 2440 \\
\hline
\end{tabular}

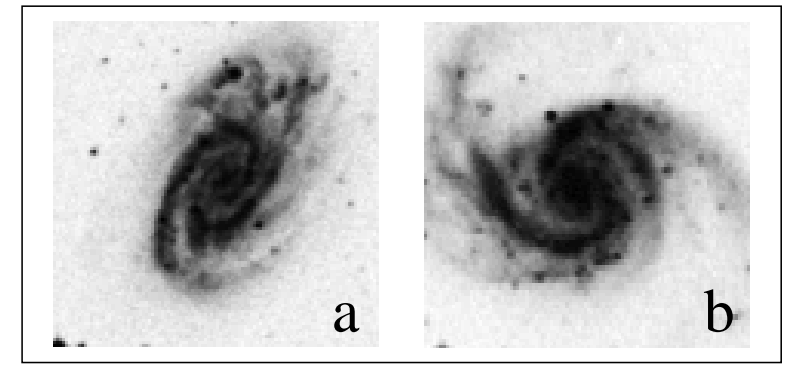

Fig. 1. Images in $B$ filter of the two studied galaxies. NGC 5861 (left) and NGC 7412 (right). North at the top, east to the left. The sides of the boxes are $3^{\prime}$

Table 2. The filters used in the observations

\begin{tabular}{lll}
\hline Filter & $\lambda(\mu \mathrm{m})$ & $\Delta \lambda(\mu \mathrm{m})$ \\
\hline$K^{\prime}$ & 2.15 & 0.32 \\
BP5 $\left(\mathrm{H}_{2}\right)$ & 2.121 & 0.039 \\
BP8 $(\mathrm{Br} \gamma)$ & 2.164 & 0.037 \\
\hline
\end{tabular}

\section{Data}

The galaxies were observed with the MPI/ESO $2.2 \mathrm{~m}$ telescope at La Silla with the broad- and narrow-band filters given in Table 2.

Exposures were obtained with the IRAC-2b camera equipped with a $256 \times 256$ pixel NICMOS-3 array (Moorwood et al. 1992; Lidman et al. 1997). All observations discussed in this paper were obtained during June 1998, except for the $K^{\prime}$ image of NGC 5861, where we used the corresponding image from Grosbøl \& Patsis (1998).

For the galaxies we observed the central $2 ! 1 \times 2 ! 1$ area, which in all cases includes the main spirals. The total ontarget integration time in seconds for each filter is given in Table 3. In parentheses we give successively, the number of integrations in the frame and the individual integration time in seconds. The target fields were interleaved with similar sky exposures in different directions. Dome flatfields were used to correct for the relative sensitivity. The median of typically four nearby sky frames was subtracted from each target exposure before flat field correction was done. Objects in the individual frames were used to derive relative offsets within a stack. Stellar images in the frames with the galaxies were visually identified and removed by fitting 2D Gaussian profiles, which then were subtracted. The average seeing during the observations was $1^{\prime \prime}$.

Table 3. Total on-target integration time in seconds for the galaxies in each filter. The numbers in the parentheses are explained in the text

\begin{tabular}{cccc}
\hline Galaxy & $K^{\prime}$ & $\mathrm{H}_{2}$ & $\mathrm{Br} \gamma$ \\
\hline NGC 5861 & $900(90,2)$ & $720(1,120)$ & $1440(1,120)$ \\
NGC 7412 & $1320(3,20)$ & $720(1,120)$ & $720(1,120)$ \\
NGC 4603 & $1440(6,20)$ & $1440(1,120)$ & $1440(1,120)$ \\
\hline
\end{tabular}




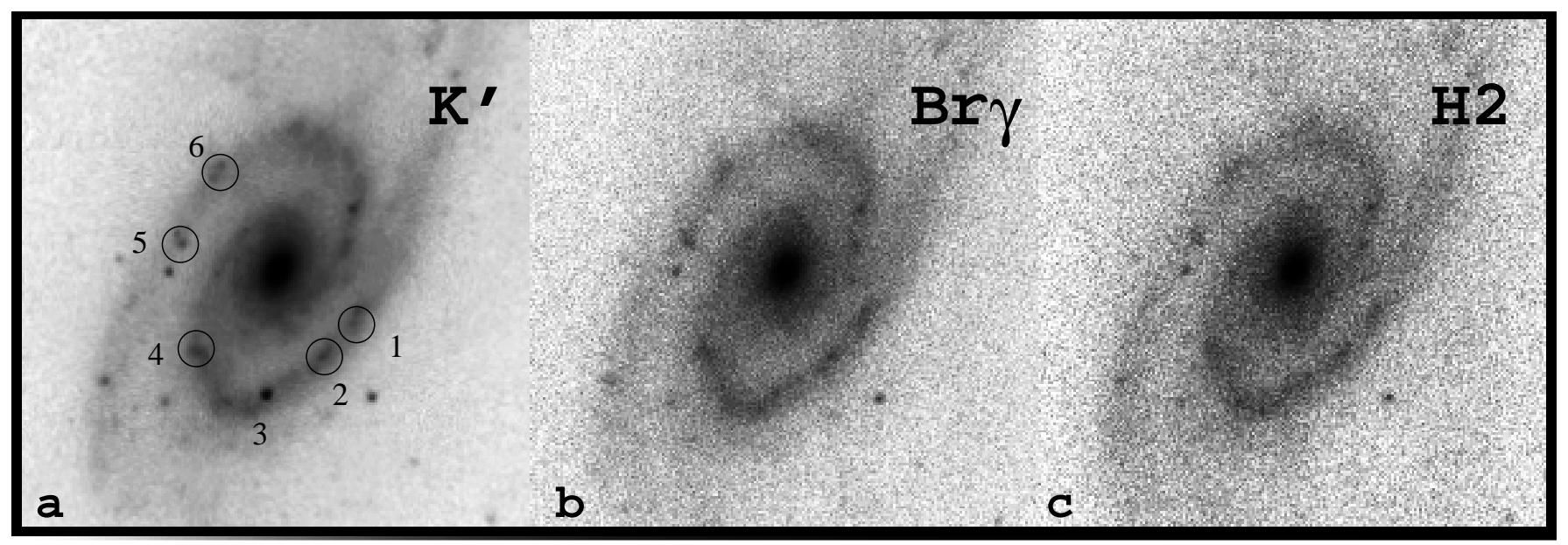

Fig. 2. NGC 5861 in $K^{\prime}$ a) and in the two narrow-band filters. $\operatorname{Br} \gamma(\mathrm{BP} 8)$ b), and $\mathrm{H}_{2}$ c). Characteristic sharp features along the arms are indicated by circles and are labeled in a). Their spatial extent indicates that they are related to extreme Pop. I objects. Images are given in logarithmic scale. North at the top, east to the left

\section{Data analysis}

\subsection{NGC 5861}

NGC 5861 offers a typical example of a galaxy with sharp features along the arms. Such a morphology characterizes the $K^{\prime}$ images of many normal spiral galaxies. In Fig. 2 we give the $K^{\prime}(\mathrm{a}), \mathrm{Br} \gamma$ (b) and $\mathrm{H}_{2}$ (c) images of the galaxy. The circles in Fig. 2a indicate the brightest sharp features on the arms; they are labeled with numbers from 1 to 6 . All labeled regions in Fig. 2a can be identified in $\mathrm{Br} \gamma$ and $\mathrm{H}_{2}$. Region "3" corresponds to a very bright sharp feature on the arms, which however cannot be studied due to a foreground star projected very close to it.

In order to normalize the narrow-band filter images to the $K^{\prime}$ one, we multiply them by a scale factor that brings the bulge-plus-interarm regions to the same level in both filters. These regions are expected to be almost free of extreme Pop. I objects and can be used for calibration purposes. As "spirals" we considered the regions with an excess of light above the axisymmetric disk at a given radius, while as "bulge" we assumed the area inside which the oval distortions at the centers of both galaxies extend, as can be seen from a radial plot of the phases of their bisymmetric components. From the bulge-plus-interarm region we excluded the central $4^{\prime \prime}$ of the galaxy to avoid possible emission from infalling gas. However, inclusion of the central region does not affect our results.

In order to estimate the contribution of $\mathrm{Br} \gamma$ and $\mathrm{H}_{2}$ in the $K^{\prime}$ light at the labeled regions we compare the excess of light in $\mathrm{Br} \gamma$ and in $\mathrm{H}_{2}$ with respect to a nearby (and at the same radial distance) arm region without a sharp feature. For this we use two images, i.e. one in $K^{\prime}$ and one in an emission line filter multiplied by a scale factor, so that the bulge-plus-interarm regions are at the same level. The use of the nearby regions at the same radial distance allows us to take into consideration the local variations in surface brightness of the galaxy, which are expected to be similar in the three filters outside the HII region. Then we subtract the flux of the "old arm region" from the flux on the sharp feature in the narrow-band filter and we normalize by the flux on the sharp feature in $K^{\prime}$. We call the resulting quantity "excess"; " $E_{\mathrm{Br} \gamma}$ " and " $E_{\mathrm{H}_{2}}$ " for the $\mathrm{Br} \gamma$ and $\mathrm{H}_{2}$ emission respectively. For $\mathrm{Br} \gamma$ e.g. it can be written symbolically:

$E_{\mathrm{Br} \gamma}=\frac{F_{\mathrm{Br}}^{\mathrm{on}}-F_{\mathrm{Br}}^{\mathrm{off}}}{F_{\mathrm{Kp}}^{\mathrm{on}}}$

where $F_{\mathrm{Br}}^{\mathrm{on}}$ and $F_{\mathrm{Br}}^{\mathrm{off}}$ are the normalized fluxes of $\mathrm{Br} \gamma$ on the sharp arm feature and off the sharp feature respectively, and $F_{\mathrm{Kp}}^{\text {on }}$ is the corresponding to $F_{\mathrm{Br}}^{\mathrm{on}}$ flux in $K^{\prime}$. A similar "excess" index can be defined for the $\mathrm{H}_{2}$ filter.

In Table 4 we give for NGC 5861 the "excess" in $\mathrm{Br} \gamma$ for the regions named in the first column. The numbers correspond to the labels in Fig. 2a. In the third column we give the the $\mathrm{Br} \gamma / K^{\prime}$ ratio at the nearby out-of-the-sharpfeature arm region. Standard deviations for the measurements on the regions where we calculated the "excess" are of the order of $10 \%$ of the given value. As we see, the additional flux on the bright, sharp feature in $\operatorname{Br} \gamma$ is of the order of $20 \%$ of the $K^{\prime}$ flux in the same region.

The same quantities for the narrow-band filter $\mathrm{H}_{2}$ in the same regions are given in the subsequent columns. The "excess" in this case is found to be systematically smaller

Table 4. NGC 5861. The columns show the label of the sharp feature, its radial distance on the deprojected image of the galaxy in arcsec, the $\mathrm{Br} \gamma$ excess, the $\mathrm{Br} \gamma / K^{\prime}$ ratio at the nearby region, the $\mathrm{H}_{2}$ excess and the $\mathrm{H}_{2} / K^{\prime}$ ratio

\begin{tabular}{cccccc}
\hline region & $R$ & $E_{\mathrm{Br} \gamma}$ & $\mathrm{Br} \gamma / K^{\prime}$ & $E_{\mathrm{H}_{2}}$ & $\mathrm{H}_{2} / K^{\prime}$ \\
\hline 1 & 31.5 & 0.15 & 1.00 & 0.15 & 1.01 \\
2 & 27.8 & 0.21 & 1.03 & 0.12 & 1.01 \\
4 & 22.8 & 0.23 & 1.00 & 0.14 & 1.02 \\
5 & 34.3 & 0.27 & 1.00 & 0.22 & 1.00 \\
6 & 49.7 & 0.26 & 0.99 & 0.16 & 1.00 \\
\hline
\end{tabular}




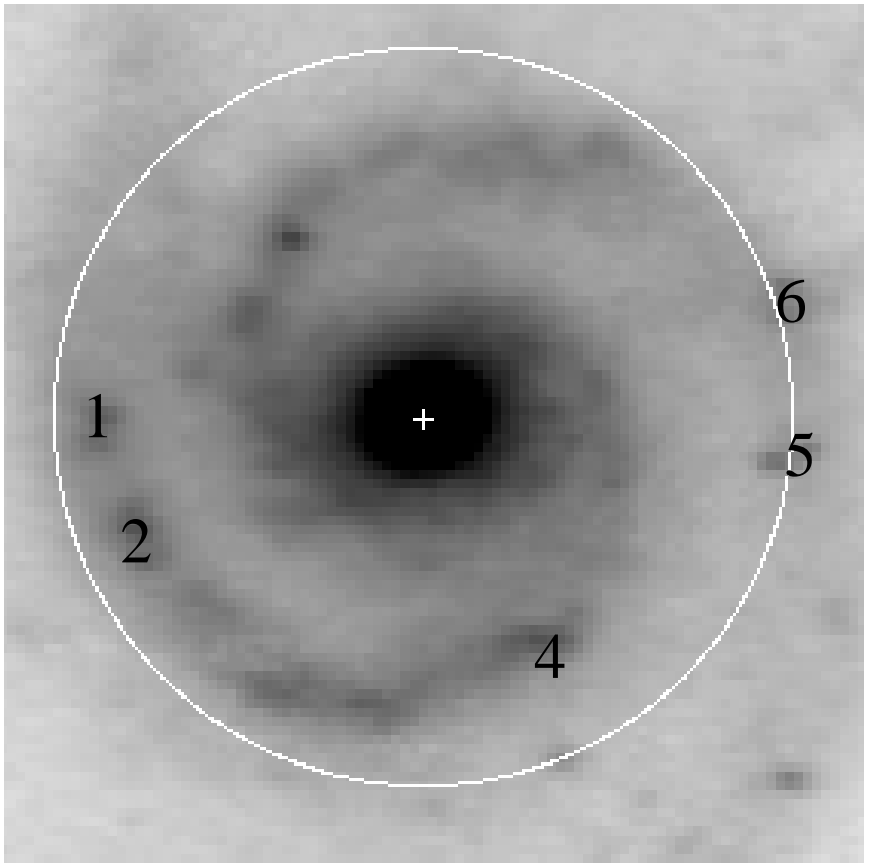

Fig. 3. Deprojected $K^{\prime}$ image of NGC 5861. Regions "5" and "6" have the largest galactocentric distance

and is of the order of $15 \%$. In both cases region " 5 " seems to be relatively more important. Feinstein (1997) characterizes all regions labeled in Fig. 2a as HII regions. The size of an HII region does not strongly depend on radius, while the old population disk falls exponentially. On a deprojected image of this galaxy (Fig. 3) we note that regions "5" and " 6 " are those with the largest galactocentric distance. Thus, their relative largest brightness is to be expected. Nevertheless, the difference is small due to the fact that the spirals of NGC 5861 have a small pitch angle about $12^{\circ}$ (Grosbøl \& Patsis 1998). Hence, the differences of the radial distances of the HII regions are in general small.

\subsection{NGC 7412}

The HII regions in NGC 7412 have been mapped by Saraiva-Schröder et al. (1994). In Table 5 we give the $\mathrm{Br} \gamma$ and $\mathrm{H}_{2}$ "excesses" as we do in Table 4 for NGC 5861. We keep the same numbering for characterizing the knotty features on the arms as in Fig. 7 of Saraiva-Schröder et al. (1994). Here we find a less peaked distribution of $E_{\operatorname{Br} \gamma}$. In comparison to NGC 5861. The mean value however is again about 0.2 .

\section{Contribution of line emission}

The main correction one has to do in order to estimate the contribution of the line emission from the narrow band filter to the total light in $K^{\prime}$ is due to the ratio of the effective widths of the two filters. Certainly, population effects may change the flux level at a given wavelength
Table 5. NGC 7412. The columns show the label of the sharp feature, the $\mathrm{Br} \gamma$ excess, the $\mathrm{Br} \gamma / K^{\prime}$ ratio at the nearby region, the $\mathrm{H}_{2}$ excess and the $\mathrm{H}_{2} / K^{\prime}$ ratio

\begin{tabular}{ccccc}
\hline region & $E$ & $\mathrm{Br} \gamma / K^{\prime}$ & $E_{\mathrm{H}_{2}}$ & $\mathrm{H}_{2} / K^{\prime}$ \\
\hline 2 & 0.45 & 1.00 & 0.20 & 0.95 \\
5 & 0.37 & 1.00 & 0.23 & 0.95 \\
19 & 0.09 & 0.99 & 0.02 & 1.01 \\
20 & 0.15 & 1.01 & 0.13 & 1.06 \\
23 & 0.15 & 1.01 & 0.05 & 0.95 \\
$3+4$ & 0.21 & 1.06 & 0.21 & 1.07 \\
\hline
\end{tabular}

in the broad filter $K^{\prime}$. Also, HII regions are powered by OB stars, which also emit continuum flux.

We consider two images, one in $K^{\prime}$ and the other one in a narrow-band filter, e.g. BP8 $(\operatorname{Br} \gamma)$. The flux measured in $K^{\prime}$ is:

$F_{K^{\prime}}=\int_{0}^{\infty} T_{K^{\prime}}(\lambda)\left\{S_{\mathrm{o}}(\lambda)+S_{y}(\lambda)+\ell_{\mathrm{Br} \gamma}+\ell_{\mathrm{H}_{2}}\right\} \mathrm{d} \lambda$

where $T_{K^{\prime}}$ is the transmission of the $K^{\prime}$ filter, $S_{\text {o }}$ refers to the continuum of the old stellar population, $S_{y}$ to the young stellar population and $\ell_{x}$ to the contributions of the emission lines.

The flux measured in a narrow-band filter, e.g. Br $\gamma$ on the other hand is:

$F_{\mathrm{Br} \gamma}=\int_{0}^{\infty} T_{\mathrm{BP} 8}(\lambda)\left\{S_{\mathrm{o}}(\lambda)+S_{y}(\lambda)+\ell_{\mathrm{Br} \gamma}\right\} \mathrm{d} \lambda$

where $T_{\mathrm{BP} 8}$ is the transmission of the $\mathrm{BP} 8$ filter.

In order to estimate these fluxes, we assume $S_{\mathrm{o}}$ and $S_{y}$ constant, and $\ell_{x}$, i.e. the line emissions, to be delta functions. Under these assumptions and using $T_{\mathrm{Br} \gamma}=$ $T_{\mathrm{BP} 8}\left(\lambda_{\mathrm{Br} \gamma}\right)$, Eqs. (2) and (3) can be written as:

$F_{K^{\prime}}=w_{K^{\prime}} S_{\mathrm{o}}+w_{K^{\prime}} S_{y}+\left.T_{K^{\prime}}\right|_{\lambda_{B}} \ell_{\mathrm{Br} \gamma}+\left.T_{K^{\prime}}\right|_{\lambda_{\mathrm{H}_{2}}} \ell_{\mathrm{H}_{2}}$

and

$F_{\mathrm{Br} \gamma}=w_{\mathrm{Br} \gamma} S_{\mathrm{o}}+w_{\mathrm{Br} \gamma} S_{y}+T_{\mathrm{Br} \gamma} \ell_{\mathrm{Br} \gamma}$

respectively,

with $w_{K^{\prime}}=\int_{0}^{\infty} T_{K^{\prime}} \mathrm{d} \lambda, w_{\mathrm{Br} \gamma}=\int_{0}^{\infty} T_{\mathrm{BP} 8} \mathrm{~d} \lambda, T_{K^{\prime}}(\lambda)$ assumed constant over the effective width of the $K^{\prime}$ filter and $\left.T_{K^{\prime}}\right|_{\lambda_{B}},\left.T_{K^{\prime}}\right|_{\lambda_{\mathrm{H}_{2}}}$ the actual transmissions at the $\operatorname{Br} \gamma$ and $\mathrm{H}_{2}$ lines respectively. Hereafter, whenever $T_{K^{\prime}}$ will be followed by $\ell_{x}$, we mean the actual transmission at the " $x$ " line.

Since our images are normalized so that they have their bulge-plus-interarm regions at the same level, the flux we measure in our narrow-band-filter image is:

$$
\begin{aligned}
F_{\mathrm{Br} \gamma}^{\prime} & =\left(w_{K^{\prime}} / w_{\mathrm{Br} \gamma}\right) F_{\mathrm{Br} \gamma} \\
& =w_{K^{\prime}} S_{\mathrm{o}}+w_{K^{\prime}} S_{y}+\left(w_{K^{\prime}} / w_{\mathrm{Br} \gamma}\right) T_{\mathrm{Br} \gamma} \ell_{\mathrm{Br} \gamma}
\end{aligned}
$$


When we measure the Br $\gamma$ "excess" we measure actually

$$
\begin{aligned}
E_{\mathrm{Br} \gamma} & =\frac{F_{\mathrm{Br}}^{\prime \text { on }}-F_{\mathrm{Br}}^{\mathrm{oof}}}{F_{K^{\prime}}^{\mathrm{on}}} \\
& \approx \frac{w_{K^{\prime}} S_{y}+\left(w_{K^{\prime}} / w_{\mathrm{Br} \gamma}\right) T_{\mathrm{Br} \gamma} \ell_{\mathrm{Br} \gamma}}{w_{K^{\prime}} S_{\mathrm{o}}+w_{K^{\prime}} S_{y}+T_{K^{\prime}} \ell_{\mathrm{Br} \gamma}+T_{K^{\prime}} \ell_{\mathrm{H}_{2}}} .
\end{aligned}
$$

In a case where the only contribution from young objects would be due to the extreme Pop. I continuum, i.e. for galaxies in which the line is shifted out of the narrow-band filter due to their large systemic velocity, the measured "excess" would be:

$E_{\mathrm{Br} \gamma}=\frac{w_{K^{\prime}} S_{y}}{w_{K^{\prime}} S_{\mathrm{o}}+w_{K^{\prime}} S_{y}+T_{K^{\prime}} \ell_{\mathrm{Br} \gamma}+T_{K^{\prime}} \ell_{\mathrm{H}_{2}}}$.

In this case, measuring the excess, we get the relative continuum flux of young objects. However, the ratio we want to know includes also the line emissions. It is:

$r=\frac{w_{K^{\prime}} S_{y}+T_{K^{\prime}} \ell_{\mathrm{Br} \gamma}+T_{K^{\prime}} \ell_{\mathrm{H}_{2}}}{w_{K^{\prime}} S_{\mathrm{o}}+w_{K^{\prime}} S_{y}+T_{K^{\prime}} \ell_{\mathrm{Br} \gamma}+T_{K^{\prime}} \ell_{\mathrm{H}_{2}}}$.

In order to estimate the contribution of the individual lines, one has first to subtract from $r$ the contribution of the young continuum and then to estimate the $T_{K^{\prime}} \ell_{x} / F_{K^{\prime}}^{\text {on }}$ ratios. However, these are minor contributions. This can be seen from Eq. (7). Let us assume for the sake of the argument that the total measured flux was just due the flux of one line. So in the numerator of Eq. (7) would appear only the quantity $\left(w_{K^{\prime}} / w_{\mathrm{Br} \gamma}\right) T_{\operatorname{Br} \gamma} \ell_{\mathrm{Br} \gamma}$. Then, first we have to divide this numerator by $\left(w_{K^{\prime}} / w_{\mathrm{Br} \gamma}\right)$ in order to get $T_{\mathrm{Br} \gamma} \ell_{\mathrm{Br} \gamma}$ and further we have to correct for the (small) difference between $T_{K^{\prime}}$ and $T_{\mathrm{Br} \gamma}$ (see Fig. 4) by multiplying with $T_{K^{\prime}} / T_{\mathrm{Br} \gamma}$ to get $T_{K^{\prime}} \ell_{x} / F_{K^{\prime}}^{\text {on }}$. The factor $\left(w_{K^{\prime}} / w_{\mathrm{Br} \gamma}\right)$ alone is $\approx 9$, which reduces the relative contribution of the line flux to the $K^{\prime}$ band by almost an order of magnitude.

A way to estimate the contribution of the young continuum is described in the following section.

\subsection{Continuum radiation estimate}

NGC 5861 and NGC 7412 have systemic velocities 1888 and $1717 \mathrm{~km} \mathrm{~s}^{-1}$ respectively (RC3), while most other galaxies in the sample have systemic velocities larger than $2700 \mathrm{~km} \mathrm{~s}^{-1}$. Because of this, the emission lines are redshifted out of the narrow-band $\mathrm{Br} \gamma$ and $\mathrm{H}_{2}$ filters. This does not happen in the cases of NGC 5861 and NGC 7412 as one can realize by plotting the transmission curves (Lidman et al. 1997). In Fig. 4 we give these curves for $K^{\prime}, \mathrm{Br} \gamma$ and $\mathrm{H}_{2}$. In the narrow-band filters the $\mathrm{Br} \gamma$ and $\mathrm{H}_{2}$ lines are not centered; they are shown in Fig. 4 at $\lambda=2.164$ and $\lambda=2.121 \mu \mathrm{m}$ respectively. To their right we plot also the redshifted lines for the case of NGC 5861, which has a redshift 0.0063 , i.e. the lines are shifted by $\Delta \lambda=0.013 \mu \mathrm{m}$ and $\Delta \lambda=0.012 \mu \mathrm{m}$. We observe, that there is no significant loss of flux in this case, mainly because the narrow-band filters are off-centered by construction in respect to the lines.

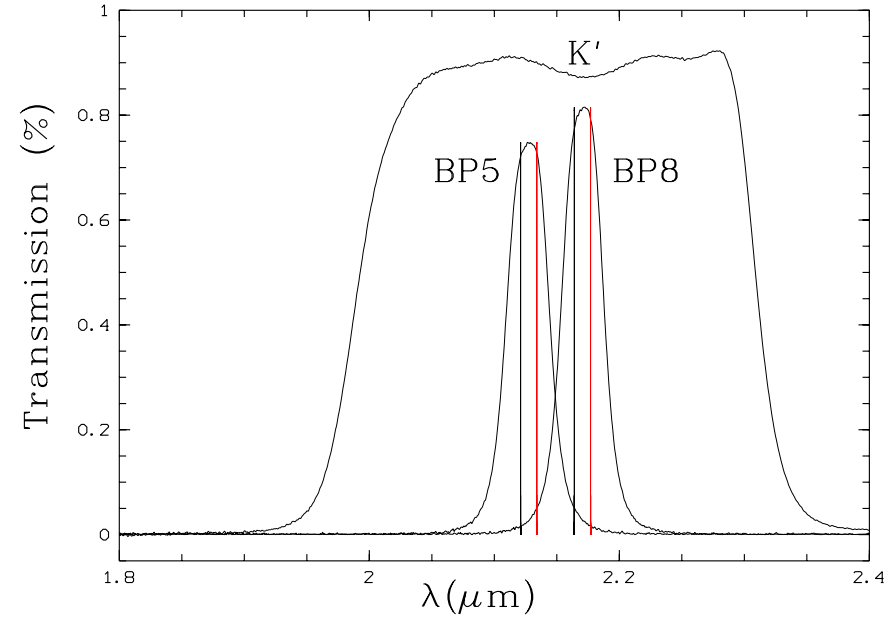

Fig. 4. The transmission curves of the broad band filter $K^{\prime}$ and the two narrow-band filters BP8 $(\mathrm{Br} \gamma)$ and BP5 $\left(\mathrm{H}_{2}\right)$ used. The lines corresponds to the original and redshifted lines for the case of NGC 5861

However, if we consider e.g. the galaxy NGC 4603 (Fig. 5), with systemic velocity $2440 \mathrm{~km} \mathrm{~s}^{-1}$ (RC3), then most of the flux is lost, as the lines are shifted to the tail of the transmission curve by $\Delta \lambda=0.0197$ and $\Delta \lambda=0.0193$ for $\mathrm{Br} \gamma$ and $\mathrm{H}_{2}$ respectively. Calculating the "excess" for the regions marked with small circles in Fig. 5 we find results of the same order as in previous cases, namely 15$20 \%$. This can be seen in Table 6 . The fact that both when the emission lines are included (Eq. (7)) and when only continuum radiation is present in the line filters (Eq. (8)), the found "excess" is of the same order, suggests that this "excess" is mainly due to an extreme Pop. I continuum.

Table 6. NGC 4603. Columns as in Table 5

\begin{tabular}{ccccc}
\hline region & $E$ & $\mathrm{Br} \gamma / K^{\prime}$ & $E_{\mathrm{H}_{2}}$ & $\mathrm{H}_{2} / K^{\prime}$ \\
\hline 1 & 0.15 & 0.98 & 0.15 & 1.00 \\
2 & 0.04 & 0.99 & 0.04 & 1.01 \\
3 & 0.04 & 0.96 & 0.02 & 0.96 \\
4 & 0.15 & 0.97 & 0.14 & 0.96 \\
5 & 0.25 & 1.00 & 0.20 & 1.00 \\
6 & 0.15 & 1.00 & 0.13 & 0.98 \\
\hline
\end{tabular}

\section{Discussion and conclusions}

We obtained for three grand design spiral galaxies with bright knots on their arms broad and narrow band nearinfrared photometry. We investigated the importance of $\mathrm{Br} \gamma$ and $\mathrm{H}_{2}$ emission in $K^{\prime}$ compared to the continuum contribution due to the presence of young objects. The excess of light on a knot of the spiral arms compared with a nearby region of the arm out of the knot was used to estimate the ratio of the line emission over the continuum. The measured "excesses" are found to be typically in the range of 0.15 to 0.3 . By using images of galaxies with the lines red-shifted almost out of the $K^{\prime}$ filter we find similar 


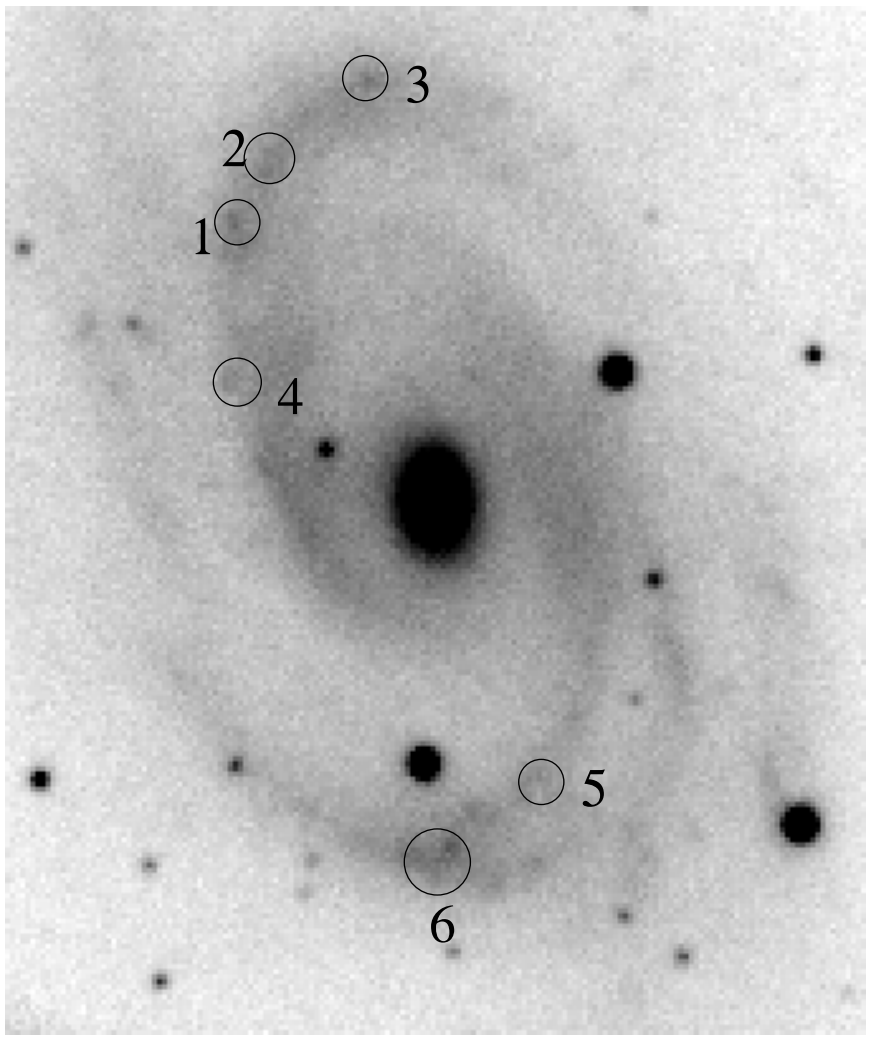

Fig. 5. NGC 4603 in $K^{\prime}$. The circles indicate the six regions mentioned in Table 6

"excesses". Thus, we conclude that the main young object contribution comes from an extreme Pop. I continuum.

Measuring "excesses" of about 0.2 , means that the line contribution can only be about a few percent and one concludes that the contribution of $\mathrm{Br} \gamma$ and $\mathrm{H}_{2}$ emission lines in the total flux emitted in $K^{\prime}$ is not important. As we can see from Eq. (7) the line contribution is drastically reduced after the correction for the relative width of the filters.

In view of this, the most important contributions from young objects in $K^{\prime}$ are the red supergiants as described in Rix \& Rieke (1993).
Acknowledgements. It is a pleasure to acknowledge fruitful discussions and valuable comments by Prof. Hans-Walter Rix and Prof. G. Contopoulos. We thank the $2.2 \mathrm{~m}$ team in La Silla and especially James Brewer and Chris Lidman for discussions about the filters. This work has been partly supported by SFB 328; by EПET II and KП $\Sigma$ 1994-1999; and by the Research Committee of the Academy of Athens.

\section{References}

Bertin, G., Lin, C. C., Lowe, S. A., \& Thurstans, R. P. 1989, ApJ, 338, 78

Block, D. L., \& Puerari, I. 1999, A\&A, 342, 627

Chiosi, C., \& Vallenari, A., in Spiral galaxies in the near IR, ed. D. Minitti, \& H. W. Rix, ESO Astrophysics Symposia (Springer) 63

Contopoulos, G., \& Grosbøl, P. 1986, A\&A, 155, 11

Contopoulos, G., \& Grosbøl, P. 1988, A\&A, 187, 83

de Vaucouleurs, G., de Vaucouleurs, A., Cowien, Jr. H. G., et al. 1991, Third reference catalogue of bright galaxies (RC3) (Springer, New York)

Feinstein, C. 1997, ApJS, 112, 29

Grosbøl, P., \& Patsis, P. A. 1998, A\&A, 336, 840

Lidman, C., Gredel, R., \& Moneti, A. 1997, IRAC2b, User Manual, v.1.5, ESO, 1997

Lin, C. C., \& Yuan, Shu F. 1969, ApJ, 155, 721

Moorwood, et al. 1992, The Messenger, 69, 61

Patsis, P. A., Contopoulos, G., \& Grosbøl, P. 1991, A\&A, 243, 373

Patsis, P. A., Hiotelis, N., Contopoulos, G., \& Grosbøl, P. 1994, A\&A, 286, 46

Patsis, P. A., Grosbøl, P., \& Hiotelis, N. 1997, A\&A, 323, 762

Rix, H. W., \& Rieke, M. 1993, ApJ, 418, 123

Rhoads, J. E. 1996, in Spiral galaxies in the near IR, ed. Minitti and Rix, ESO Astrophysics Symposia (Springer) 58

Sandage, A., \& Tammann, G. A. 1981, A Revised ShapleyAmes Catalog of Bright Galaxies (RSC), Carnegie Inst. Wash. Pub., No. 635, Washington

Saraiva-Schröder, M. F., Pastoriza, M. G., Kepler, S. O., \& Puerari, I. 1994, A\&AS, 108, 41

Toomre, A. 1981, in The structure and evolution of normal galaxies, ed. Fall, \& Lynden-Bell, 111

Wainscoat, R. J., \& Cowie, L. L. 1992, AJ, 103, 332

Wielen, R. 1977, A\&A, 60, 263 